\title{
Population Pharmacokinetic Analysis of Rivipansel in Healthy Subjects and Subjects with Sickle Cell Disease
}

\author{
Satyaprakash Nayak $^{1}$ (D) Brinda Tammara ${ }^{2}$ (D) Lutz O. Harnisch Ka $^{3,4}$
}

Accepted: 12 April 2021 / Published online: 16 May 2021

(c) The Author(s) 2021

\begin{abstract}
Background Sickle cell disease is an inherited blood disorder with reduced blood-carrying capacity. It is associated with a tendency to form microclots in blood vessels, leading to painful episodes known as a vaso-occlusive crisis. Rivipansel is a pan-selectin inhibitor being studied for the treatment of a vaso-occlusive crisis in patients with sickle cell disease.

Methods A population pharmacokinetic model of rivipansel plasma and urine concentrations was constructed using a twocompartment model and data from nine different clinical studies. Creatinine clearance was calculated using the Schwartz formula for children and the Chronic Kidney Disease Epidemiology Collaboration formula for adults. Urine volume and concentration of the study drug in urine from subjects in five clinical studies were used to estimate renal and nonrenal clearance. Results Rivipansel drug concentrations were well described by the model. The post hoc estimates of average steady-state concentrations were predicted to be similar for the adult and pediatric cohorts of the pivotal phase III study. Parameter estimates showed almost exclusively renal excretion of rivipansel, which is consistent with the known properties of the drug. Conclusions The pharmacokinetics of rivipansel was well characterized by a two-compartment population pharmacokinetic model. Our results illustrate the important role of simulations in optimizing a potential drug dosing regimen for patients with sickle cell disease and progressive renal impairment.
\end{abstract}

\section{Key Points}

Rivipansel pharmacokinetics was found to be linear

A two-compartment model with renal and nonrenal clearance explained the pharmacokinetics well

Model simulations helped in comparing the cohorts in the pivotal study in order to combine them for the primary efficacy analysis
Lutz O. Harnisch

Lutz.O.Harnisch@pfizer.com

Pfizer Inc., Cambridge, MA, USA

2 Pfizer Inc., Collegeville, PA, USA

3 Pfizer Ltd, London, UK

4 Global Clinical Pharmacology, Pfizer Inc., IPC096, Ramsgate Road, Sandwich, Kent CT13 9NJ, UK

\section{Introduction}

Sickle cell disease (SCD) affects millions of people worldwide, including approximately 100,000 people in the USA [1]. It is a chronic condition associated with substantial morbidity and mortality, and is responsible for more than 75,000 hospitalizations per year in the USA, with an average stay of more than 6 days [2]. Both children and adults are affected [3], and greater mortality is seen in those with more severe disease [3-5]. Sickle cell disease is associated with a number of serious and potentially disabling conditions that have similar symptoms but vary in severity by genotype $[4,6]$. The disorder affects the shape of red blood cells and makes them adhere to the blood vessels, leading to formation of micro-clots in blood vessels [7]. This leads to a vaso-occlusive crisis (VOC), a painful and serious consequence of SCD, presumably resulting from acute ischemic tissue injury $[7,8]$.

Rivipansel (formerly PF-06460031) was licensed by Pfizer from GlycoMimetics in 2011, and the Investigational New Drug application was transferred to Pfizer in the second half of 2013. This compound is a pan-selectin antagonist that has been shown to inhibit selectin binding and to improve 
blood flow and survival in an animal model of SCD. Selectins are known to play a major role in the adhesion of blood cells to the endothelium of blood vessels and to facilitate the interaction between sickled red blood cells, platelets, leukocytes, and endothelial cells [7, 9]. Selectin binding is a key early step in the inflammatory process, leading to leukocyte adhesion and recruitment to inflamed tissue [7].

Three previous phase I studies were completed in healthy volunteers and subjects with SCD who were not experiencing a VOC. Further phase I studies assessed the effects of age, hepatic impairment, and renal impairment on the pharmacokinetic (PK) properties of rivipansel [10]. In the phase I studies, the PK profile of rivipansel was shown to be linear and predictable, with a mean half-life of 7-8 h, no dose-related trends observed in clearance (CL) or halflife, with rivipansel almost exclusively eliminated renally. Additionally, no in vitro metabolism was observed in studies with rat, monkey, or human hepatocytes [11]. The phase I studies were followed by a randomized phase II study (NCT01119833) that evaluated the efficacy, safety, and pharmacokinetics of two dosing regimens of rivipansel in patients with SCD aged 12-60 years who were hospitalized for a VOC [12].

The PK data compiled to date reveal that rivipansel is almost completely eliminated unchanged in urine and has low potential for drug-drug interactions. This is important because hyperfiltration, an abnormal increase in the filtration rate of the renal glomeruli resulting in an elevated estimated glomerular filtration rate, has been reported in patients with
SCD [13, 14]. The majority of children with SCD exhibit hyperfiltration, but the degree of hyperfiltration depends on a number of factors, including the SCD genotype [14-17].

The pivotal phase III study of rivipansel (NCT02187003), a multicenter, randomized, double-blind, placebo-controlled, parallel-group study conducted to evaluate the efficacy and safety of rivipansel in the treatment of VOCs in hospitalized adult and pediatric patients with SCD, was recently completed. Clinical results of this study are reported elsewhere [18].

The primary objective of the current analysis was to develop a population PK model describing the pharmacokinetics of rivipansel using the combined data of all previous studies. A secondary objective was to generate post hoc estimates for average steady-state concentration $\left(C_{\mathrm{avg}, \mathrm{ss}}\right)$ for the two cohorts in the phase III study (Cohort 1: children aged $\geq 12$ years and adults; Cohort 2: children aged 6-11 years), provide a comparison between rivipansel exposure between the two cohorts, and provide a rationale for dose adjustment in renally impaired patients, if required.

\section{Methods}

\subsection{Study Population and Dosing}

A brief overview of the studies included in this analysis is shown in Table $1[12,19,20]$. A more detailed description of the studies, including dosing information, is provided in

Table 1 Overview of studies included in the analysis

\begin{tabular}{|c|c|c|}
\hline Study number/Clinicaltrials.gov identifier & Study sponsor & Description \\
\hline B5201001/NCT01941511 [19] & Pfizer & $\begin{array}{l}\text { A phase I, single-dose study to evaluate the effect of intravenous doses of rivipansel } \\
\text { on QTc intervals in } 48 \text { healthy black adult subjects }\end{array}$ \\
\hline B5201002/NCT02187003 & Pfizer & $\begin{array}{l}\text { A phase III, multiple-dose study to evaluate the efficacy and safety of rivipansel in } \\
\text { the treatment of VOC in } 300 \text { hospitalized subjects with SCD }\end{array}$ \\
\hline B5201004/NCT02998099 & Pfizer & $\begin{array}{l}\text { A phase I, single-dose study to estimate the effect of age on the PK, safety, and } \\
\text { tolerability of rivipansel in } 16 \text { subjects }\end{array}$ \\
\hline B5201005/NCT02813798 & Pfizer & $\begin{array}{l}\text { A phase I, single-dose study to estimate the effect of age of the PK, safety, and } \\
\text { tolerability of rivipansel in } 21 \text { subjects with renal impairment and } 7 \text { healthy } \\
\text { subjects }\end{array}$ \\
\hline B5201006/NCT02871570 & Pfizer & $\begin{array}{l}\text { A phase I, single-dose study to evaluate the PK, safety, and tolerability of rivipansel } \\
\text { in eight subjects with moderate hepatic impairment and in eight healthy subjects } \\
\text { with normal hepatic function }\end{array}$ \\
\hline B5201009 & Glycomimetics & $\begin{array}{l}\text { A phase I, single ascending-dose study to evaluate the safety, tolerability, and PK } \\
\text { of rivipansel in } 40 \text { healthy adult subjects }\end{array}$ \\
\hline B5201010 & Glycomimetics & $\begin{array}{l}\text { A phase I, multiple ascending-dose study to evaluate the safety, tolerability, and } \\
\text { PK of rivipansel in } 32 \text { healthy adults }\end{array}$ \\
\hline B5201011/NCT00911495 [20] & Glycomimetics & A phase I/II study of the safety and PK of rivipansel in 20 adults with SCD \\
\hline B5201012/NCT01119833 [12] & Glycomimetics & $\begin{array}{l}\text { A phase II study of the efficacy, safety, and PK of rivipansel in subjects hospital- } \\
\text { ized for SCD VOC }\end{array}$ \\
\hline
\end{tabular}

$P K$ pharmacokinetics, $S C D$ sickle cell disease, $V O C$ vaso-occlusive crisis

See Table 1 of the ESM for a more detailed description of the studies, such as subject information and dosing regimen 
Table 1 of the Electronic Supplementary Material (ESM). A summary of demographics and baseline characteristics of the population used in this analysis is provided in Table 2.

\subsection{Previous Modeling Experience}

Three internal analyses for phase I and/or phase II studies (B5201009, B5201010, B5201011 [20], B5201012 [12])

Table 2 Demographics and baseline characteristics (active treatment only)

\begin{tabular}{|c|c|c|c|c|c|}
\hline Categorical parameter, $n(\%)$ & \multicolumn{2}{|l|}{ Phase I } & Phase II & Phase III & Total \\
\hline Subjects & \multicolumn{2}{|l|}{174} & 43 & 156 & 373 \\
\hline \multicolumn{6}{|l|}{ Sex } \\
\hline Male & \multicolumn{2}{|l|}{$133(76.4)$} & $18(41.9)$ & $78(50.0)$ & $229(61.4)$ \\
\hline Female & \multicolumn{2}{|l|}{$41(23.6)$} & $25(58.1)$ & $78(50.0)$ & $144(38.6)$ \\
\hline \multicolumn{6}{|l|}{ Age category (years) } \\
\hline $6-11$ & \multicolumn{2}{|l|}{$0(0.0)$} & $0(0.0)$ & $23(14.7)$ & $23(6.2)$ \\
\hline $12-17$ & \multicolumn{2}{|l|}{$0(0.0)$} & $13(30.2)$ & $43(27.6)$ & $56(15.0)$ \\
\hline $18-64$ & \multicolumn{2}{|l|}{$151(86.8)$} & $30(69.8)$ & $90(57.7)$ & $271(72.7)$ \\
\hline$\geq 65$ & \multicolumn{2}{|l|}{$23(13.2)$} & $0(0.0)$ & $0(0.0)$ & $23(6.2)$ \\
\hline Race & & & & & \\
\hline Asian & $14(8.0)$ & & $0(0.0)$ & $0(0.0)$ & $14(3.8)$ \\
\hline Black & $62(35.6)$ & & $41(95.3)$ & $151(96.8)$ & $254(68.1)$ \\
\hline Other & $7(4.0)$ & & $2(4.7)$ & $5(3.2)$ & $14(3.8)$ \\
\hline White & $91(52.3)$ & & $0(0.0)$ & $0(0.0)$ & $91(24.4)$ \\
\hline Population & & & & & \\
\hline Non-SCD & $160(92.0)$ & & $0(0.0)$ & $0(0.0)$ & $160(42.9)$ \\
\hline SCD without VOC & $14(8.0)$ & & $0(0.0)$ & $0(0.0)$ & $14(3.8)$ \\
\hline SCD with VOC & $0(0.0)$ & & $43(100.0)$ & $156(100.0)$ & $199(53.4)$ \\
\hline Renal impairment & & & & & \\
\hline None & $7(4.0)$ & & $0(0.0)$ & $0(0.0)$ & $7(1.9)$ \\
\hline Mild & $7(4.0)$ & & $0(0.0)$ & $0(0.0)$ & $7(1.9)$ \\
\hline Moderate & $7(4.0)$ & & $0(0.0)$ & $0(0.0)$ & $7(1.9)$ \\
\hline Severe & $7(4.0)$ & & $0(0.0)$ & $0(0.0)$ & $7(1.9)$ \\
\hline Hepatic impairment & & & & & \\
\hline None & $8(4.6)$ & & $0(0.0)$ & $0(0.0)$ & $8(2.1)$ \\
\hline Moderate & $8(4.6)$ & & $0(0.0)$ & $0(0.0)$ & $8(2.1)$ \\
\hline Continuous variables & Non-SCD, adults & $\begin{array}{l}\text { SCD without } \\
\text { VOC, adults }\end{array}$ & $\begin{array}{l}\text { SDC with VOC, } \\
6-11 \text { y }\end{array}$ & $\begin{array}{l}\text { SCD with VOC, } \\
12-17 \mathrm{y}\end{array}$ & SCD with VOC, adults \\
\hline Age (y) & & & & & \\
\hline Mean (CV\%) & $41(42.6)$ & $33(33.0)$ & $10(19.0)$ & $15(12.5)$ & $28(31.3)$ \\
\hline Range & 19-80 & $19-50$ & $6-11$ & $12-17$ & $18-56$ \\
\hline Weight (kg) & & & & & \\
\hline Mean (CV\%) & $80(17.7)$ & $65(15.7)$ & $33(35.3)$ & $53(25.7)$ & $70(22.5)$ \\
\hline Range & $49-131$ & 48-91 & $18-65$ & $31-81$ & $41-145$ \\
\hline Height (cm) & & & & & \\
\hline Mean (CV\%) & $173(5.7)$ & $171(3.2)$ & $136(9.4)$ & $159(7.5)$ & $168(5.6)$ \\
\hline Range & $141-198$ & $162-180$ & $109-158$ & $136-188$ & $140-196$ \\
\hline Serum creatinine $(\mathrm{mg} / \mathrm{dL})$ & & & & & \\
\hline Mean (CV\%) & $1.1(57.1)$ & $0.7(36.1)$ & $0.4(27.7)$ & $0.5(25.0)$ & $0.7(29.8)$ \\
\hline Range & $0.5-4.6$ & $0.4-1.4$ & $0.2-0.7$ & $0.3-0.8$ & $0.2-1.6$ \\
\hline Creatinine clearance $(\mathrm{mL} / \mathrm{min}$ & & & & & \\
\hline Mean (CV\%) & $111.3(31.7)$ & $119.5(23.6)$ & $91.6(32.5)$ & $118.7(27.6)$ & $144.7(19.9)$ \\
\hline Range & $14.8-180.7$ & $64.2-167.5$ & $49.9-163.1$ & $69.4-203.7$ & $70.9-214.8$ \\
\hline
\end{tabular}

$C V \%$ coefficient of variance $\%, S C D$ sickle cell disease, $V O C$ vaso-occlusive crisis, $y$ years 
were performed. In the first analysis, which included studies B5201009, B5201010, and B5201011, two-compartment and three-compartment linear PK models (implemented in nonlinear mixed-effects modeling using NONMEM Version 7.1.2 [ICON plc, Dublin, Ireland]) were fit to the data; the three-compartment model was preferred based on the statistical analyses. Various approaches to scaling for body size were evaluated; an allometric model was preferred statistically. No other covariates appeared to influence the PK characteristics of rivipansel. Subjects with stable SCD (study B5201011) did not appear to differ from those in the other two studies. In the second internal analysis, parameters obtained from modeling analysis 1 were used in the population PK modeling of data from the phase II study in subjects with SCD experiencing a VOC (study B5201012). The structural parameters were fixed at the values obtained from the modeling of phase I healthy volunteer data (studies B5201009 and B5201010; no longer normalized allometrically), and the magnitude of inter-individual variability was estimated. An additional factor for systemic CL was needed to reconcile the legacy values to those obtained in subjects with VOC. One covariate, creatinine clearance $(\mathrm{CrCl})$ normalized to body surface area (BSA), was added to the model.

For the third internal analysis, an integrated exercise using a nonlinear mixed-effects modeling approach (NONMEM Version 7.4) for the combined data from the phase I and II studies (B5201009, B5201010, B5201011, and B5201012) was performed. While the previous population PK model analyzed the healthy volunteer and patient trials (phase I and phase II data) independently, the combined model integrated fixed effects across all subjects who had received rivipansel. To better support extrapolation into the target population of children aged 6-11 years, the already described fixed-effects parameters and inter-individual variances were re-estimated in the previously described threecompartmental model. In the combined model using phase I and phase II study data, CL was described as a function of $\mathrm{CrCl}$, the volume of distribution was described as a function of weight, and an additional factor that distinguished CL in subjects with SCD with VOC with that estimated in healthy volunteers or subjects with stable SCD was estimated. These results were published, and the simulation results used for the dose recommendation in the population of subjects aged 6-11 years [11]. A key insight gained from the combined model was that, in general, the estimates of $\mathrm{CL}$ and volume of distribution from the combined model were not very different from the previously described individual models. However, because this model had the advantage of combining both phase I and phase II PK data to date (studies B5201009, B5201010, B5201011 and B5201012) and estimating covariances, it was used as the starting point of the current population PK modeling exercise.

\subsection{Model Description}

The base model structure was derived from the integrated analysis of phase I and phase II studies [11]. As mentioned before, it was a three-compartment model with BSA normalized $\mathrm{CrCl}$ as a covariate for $\mathrm{CL}$ and weight added as a structural covariate for the volume of distribution. Additionally, to account for hyperfiltration, an additional factor was added to CL for subjects with SCD actively experiencing a vaso-occlusive event. The Cockroft-Gault equation was used to calculate $\mathrm{CrCl}$ in the model. We employed this model to the updated dataset containing PK data from all studies listed in Table 1, except the phase III study (B5201002). We used this approach as the phase III study was still ongoing during model development. Therefore, our strategy was to develop the model without using phase III data and compare the empirical Bayesian estimates obtained from the final model with observed PK concentrations in Study B5201002 to assess the appropriateness of the model to the new (phase III) data. However, the base model with three compartments was unable to converge with the addition of new studies. Therefore, we started with a two-compartment model but also included urine data collected in a subset of studies as described in the later section.

Inter-individual variability (IIV) of the PK parameters was incorporated using a log-normal random-effects model of the form:

$\theta_{i}=\theta_{\mathrm{TV}} \times e^{\eta_{i}}$,

where $\theta_{i}$ is the individual value of the parameter (e.g., CL or volume), $\theta_{\mathrm{TV}}$ is the typical value of the parameter, and $\theta_{i}$ denotes the inter-individual random effect accounting for the $i$ th individual's deviation from the typical value. The $\eta_{i}$ were assumed to be normally distributed, with a mean of zero and a variance of $\omega^{2}$.

Renal fraction of total CL was modeled to be dependent on $\mathrm{CrCl}$ calculated by the Chronic Kidney Disease Epidemiology Collaboration (CKD-EPI) formula [21], normalized by the BSA for adults, instead of the Cockroft-Gault equation used in previous models. For children aged younger than 18 years, $\mathrm{CrCl}$ was calculated by the bedside Schwartz formula, normalized by BSA [22]. Body surface area was calculated using the Dubois and Dubois formula [23]. We tried other commonly used formulae for calculating $\mathrm{CrCl}$, such as Modification of Diet in Renal Disease [24] and the Cockroft-Gault [25], for the adult population, but we found 
that $\mathrm{CrCl}$ calculated using CKD-EPI provided the best fit for our data. Thus, renal CL was modeled as:

$\mathrm{Cl}_{i}=\theta_{\mathrm{Cl}} \times e^{\eta_{\mathrm{Cl}}} \times F_{\mathrm{CrCl}_{i}} \times F_{\mathrm{Dis}_{i}}$,

where $\theta_{\mathrm{Cl}}$ is the fixed effect for CL and $\eta_{\mathrm{Cl}}$ is the IIV for $\mathrm{CL}$, and

$F_{\mathrm{CrCl}_{i}}=\left(\frac{\mathrm{CrCl}_{i}}{\mathrm{CrCl}_{\text {median }}}\right)^{\theta_{\mathrm{CrCl}}}$,

where $F_{\mathrm{CrCl}_{i}}$ is the factor based on $\mathrm{CrCl}$. The $\mathrm{CrCl}_{\text {median }}$ is the median $\mathrm{CrCl}$ based on all the included studies, $\theta_{\mathrm{CrCl}}$ is the factor for $\mathrm{CrCl}$ dependent factor on $\mathrm{CL}$, and $F_{\mathrm{Dis}_{i}}$ is the fractional difference of change in CL based on the disease status, i.e.,

$F_{\operatorname{Dis}_{i}}=\left(1+\theta_{\operatorname{Dis}_{i}}\right)$,

where $\theta_{\operatorname{Dis}_{i}}$ is the relative increase in CL due to disease status (i.e., whether it is an SCD subject experiencing a VOC). Similarly, volume in the central and peripheral compartment was modeled as:

$\theta_{i}=\theta_{\mathrm{TV}} \times e^{\eta_{i}} \times F_{\mathrm{WT}_{i}}$,

where $F_{\mathrm{WT}_{i}}$ is the factor based on weight, defined as:

$F_{\mathrm{WT}_{i}}=\left(\frac{\mathrm{WT}_{i}}{\mathrm{WT}_{\text {median }}}\right)^{\left(\theta_{\mathrm{WT}}\right)}$.

Residual variability, a composite measure of assay error, dose/sample time collection errors, model misspecification, and any other variability was modeled using a combined (proportional and additive) error model as follows:

$Y_{i, j}=C_{i, j}+\sqrt{C_{i, j}^{2}+\theta^{2}} \times \varepsilon_{i, j}$,

where $Y_{i, j}$ denotes the observed concentrations for the $i$ th individual at the time $t_{j}, C_{i, j}$ denotes the corresponding predicted concentration based on the PK model, $\theta$ is the residual error, and $\varepsilon_{i, j}$ denotes the intra-individual (residual) random effect. The random effect was assumed to have a normal distribution with a zero mean and variance of $\sigma^{2}$. The model code is provided in the ESM.

\subsection{Incorporation of Urine Data}

Among the studies listed in Table 1, five (B5201005, B5201009, B5201010, B5201011, and B5201012) included urine PK data in addition to the plasma PK concentrations.
These studies included the volume of urine collected during a fixed time interval, (e.g., $96 \mathrm{~h}$ after dosing for the renal impairment study, B5201005), and the concentration of the drug in urine. We incorporated these data in the population PK model to calculate the renal and nonrenal fractions of total CL. We incorporated the urine data according to a recent NONMEM tutorial published by Bauer [26].

\subsection{Covariate Analysis}

The CKD-EPI formula contains age, sex, serum creatinine, and race as its inputs, and the BSA calculation by Dubois and Dubois formula requires height and weight as its inputs. Therefore, these variables were not included explicitly as covariates in the model. We explored an additional effect of weight on CL and volume by explicitly adding it as a covariate. However, the decrease in the objective function value was not significant to include weight as a covariate in the model for CL.

\subsection{Adjustment for Low CrCl Values}

Exploration of the random effects in the base model showed that the effect of $\mathrm{CrCl}$ on the random effect on $\mathrm{CL}\left(\eta_{i}\right)$ was skewed in the lower range of $\mathrm{CrCl}$ values. $\mathrm{As} \mathrm{CrCl}$ was a critical factor influencing renal $\mathrm{CL}$ of rivipansel, an additional factor was added for $\mathrm{CrCl}$ values below $60 \mathrm{~mL} / \mathrm{min}$. Thus, the final model contained the following equation for renal portion of total CL:

If $\mathrm{CrCl}$ was at least $60 \mathrm{~mL} / \mathrm{min}$ :

$F_{\mathrm{CrCl}_{i}}={\frac{\mathrm{CrCl}}{\mathrm{CrCl}_{\text {median }}}}^{\left(\theta_{1}\right)}$,

otherwise:

$F_{\mathrm{CrCl}_{\mathrm{i}}}={\frac{\mathrm{CrCl}}{\mathrm{CrCl}_{\text {median }}}}^{\left(\theta_{1}+\theta_{2}\right)}$

where $\theta_{2}$ is the additional exponent to correct for bias in $\eta_{\mathrm{CL}}$ for low $\mathrm{CrCl}$ values.

\subsection{Half-Life Calculation}

The population estimated CL was used to compare the population terminal half-life for a two-compartment model with the previously estimated values. The formula used to calculate the model-derived terminal half-life [27] is

$t_{1 / 2}=\frac{\log (2)}{1 / 2 \times\left[\left(K_{12}+K_{21}+K_{10}\right)-\sqrt{\left(K_{12}+K_{21}+K_{10}\right)^{2}-4 K_{21} K_{10}}\right]}$, 

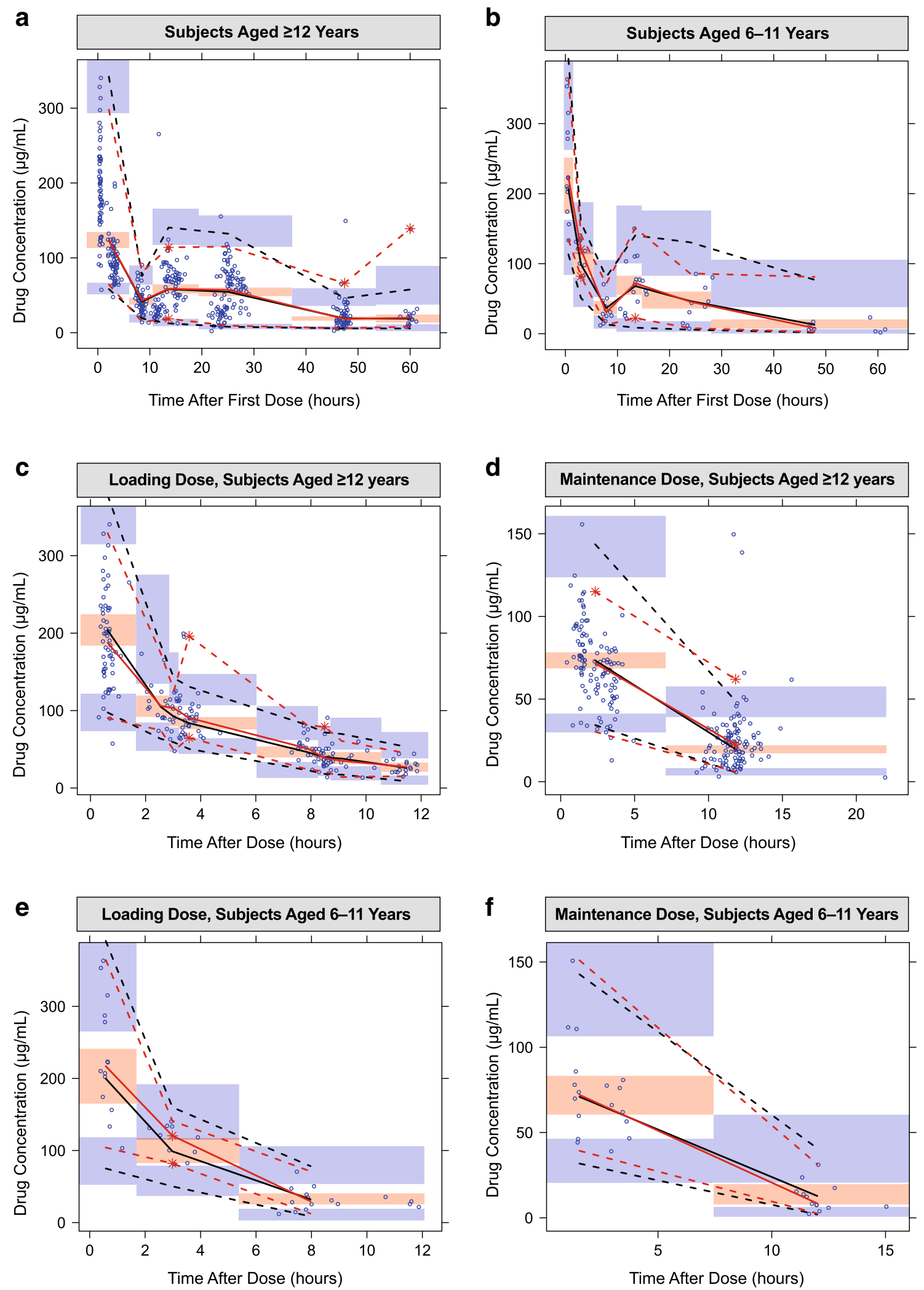
4Fig. 1 Visual predictive check (VPC) for the phase III study. The top row shows VPC plots stratified by cohorts of the phase III study, i.e., a shows subjects aged $\geq 12$ years (Cohort 1 ) and b shows subjects aged 6-11 years (Cohort 2). The middle and bottom rows show VPCs for Cohort 1 and Cohort 2 separately, stratified by loading dose and maintenance doses respectively, i.e., c shows the loading dose in subjects aged $\geq 12$ years, $\mathbf{d}$ shows the maintenance dose in subjects aged $\geq 12$ years (Cohort 1 ). Similarly, e shows the loading dose in subjects aged 6-11 years, and $\mathbf{f}$ shows the maintenance dose in subjects aged 6-11 years (Cohort 2). Note that while the $x$-axis for the top row shows time after the first administered dose, the $x$-axis for the middle and bottom rows shows time after the last administered dose

where $K_{10}=\mathrm{CL} / V_{\text {central }}, \quad K_{12}=Q / V_{\text {central }}, \quad$ a nd $K_{21}=Q / V_{\text {peripheral }}$.

\subsection{Software and Estimation Method}

The plasma and concentration-time data were analyzed using a nonlinear mixed-effects modeling methodology as implemented in the software program NONMEM Version 7.4.1 [28]. Exploratory analyses, diagnostic plots, and postprocessing of NONMEM output were performed using $\mathrm{R}$ (Version 3.6.1; R Foundation for Statistical Computing, Vienna, Austria) [29]. Perl-speaks-NONMEM (PsN) Version 4.8.0 was used for performing visual predictive checks (VPCs). The NONMEM analyses were conducted using the first-order conditional estimation method with interaction.

\subsection{Simulation Scheme}

The purpose of the simulation exercise was to assess and contrast the model-predicted $C_{\text {avg,ss }}$ value with the empirical Bayesian estimates obtained from the observed concentration data in the phase III study, B5201002. In the simulation exercise, weight was simulated from a normal distribution, with a mean and standard deviation obtained using all of the included studies for adults. For pediatric subjects, such distribution was calculated using the phase II study, which had younger subjects with SCD. The other covariate required for simulations was serum creatinine, which was simulated from a normal distribution obtained from subjects with SCD undergoing a VOC only, i.e., using the phase II study only. The design of the simulation study was similar to the phase III trial and 200 such studies were simulated in NONMEM with the model parameter values set at their final estimates. Thus, individual CLs were calculated for each simulated subject, based on their demographic data, serum creatinine, and dose of rivipansel received, which were then used to calculate simulated $C_{\mathrm{avg}, \mathrm{ss}}$.

\section{Results}

Figure 1 shows the VPC plots of rivipansel plasma concentrations from study B5201002 stratified by age cohort (Fig. 1a, b), loading dose vs maintenance doses in Cohort 1 of B5201002, subjects aged $\geq 12$ years (Fig. 1c, d), and loading dose vs maintenance doses in Cohort 2, subjects aged 6-11 years (Fig. 1e,f). The goodness-of-fit plots for rivipansel plasma concentrations for all of the included studies from the final model are provided in Fig. 1 of the ESM. As seen from the VPC, the model provided an overall good fit to the observed PK data of both the cohorts of Study B5201002 through the entire time course of treatment. Goodness-of-fit plots for all the urine concentrations in the model are shown in Fig. 2 of the ESM. The diagnostic plots of post hoc empirical Bayesian estimates of individual subjects in study B5201002 are shown in Fig. 3 of the ESM.

Table 3 shows the parameter estimates and nonparametric bootstrap results of the final model. Bootstrap analysis was performed with 1000 samples using PsN-4.8.0 software. Table 3 shows that the NONMEM estimate and the bootstrap median for the parameters match well with each other, and the residual square error is low for most parameters. The highest residual square error of about $50 \%$ is observed for the disease state-dependent hyperfiltration parameter. This could be attributed to the fact that only one study out of nine included the population experiencing an active VOC. The 95\% confidence interval obtained from the bootstrap analysis shows that most of the observed parameter estimates were tightly constrained.

Renal CL was utilized to estimate the percentage of drug eliminated in urine, and the calculated urinary excretion rates were compared with the observed values, wherever clinical data were available. Figure 2 shows a comparison between the observed and model estimated cumulative percentage of drug cleared in urine for the study with renally impaired subjects (study B5201005). Excretion rates for subjects in study B5201005 are shown in Fig. 4 of the ESM. As seen from Fig. 2, the model derived cumulative drug eliminated in urine matched well with the observed values for each cohort representing subjects with different degrees of renal impairment. A key point to note is that the observed cumulative drug eliminate in urine for the cohort with no renal impairment is calculated to be lower than nearly $100 \%(\sim 77 \%)$ owing to missing data in one 
Table 3 Parameter estimates and bootstrap results of the final pharmacokinetic model

\begin{tabular}{|c|c|c|c|c|}
\hline Parameter & Estimate & $\begin{array}{l}\text { Relative standard } \\
\text { error }(\%)\end{array}$ & Bootstrap median & Bootstrap 95\% CI \\
\hline $\mathrm{CL}(\mathrm{L} / \mathrm{h})$ & 1.15 & 1.91 & 1.15 & $1.10-1.19$ \\
\hline $\mathrm{CL}_{\mathrm{n}}(\mathrm{L} / \mathrm{h})$ & 0.0718 & 22.2 & 0.0686 & $0.0380-0.108$ \\
\hline$V_{\mathrm{c}}(\mathrm{L})$ & 6.75 & 2.56 & 6.72 & $6.40-7.06$ \\
\hline$Q(\mathrm{~L} / \mathrm{h})$ & 2.01 & 6.60 & 2.02 & $1.80-2.25$ \\
\hline$V_{\mathrm{p}}(\mathrm{L})$ & 4.48 & 2.80 & 4.49 & $4.27-4.74$ \\
\hline Fractional increase in CL for SCD-VOC subjects & 0.116 & 50.4 & 0.115 & $0.0204-0.220$ \\
\hline $\mathrm{CrCl}$ factor exponent on $\mathrm{CL}$ & 0.477 & 12.8 & 0.471 & $0.349-0.594$ \\
\hline Additional additive exponent at low $\mathrm{CrCl}(<60 \mathrm{~mL} / \mathrm{min})$ & 0.413 & 17.6 & 0.412 & $0.263-0.564$ \\
\hline Weight factor exponent on $V_{\mathrm{c}}$ & 0.512 & 14.4 & 0.511 & $0.368-0.664$ \\
\hline IIV, CL (CV\%) & 20.0 & 16.4 & 20.0 & $16.7-23.7$ \\
\hline Correlation, CL and $V_{\mathrm{c}}$ & 0.513 & 37.6 & 0.535 & $0.320-0.703$ \\
\hline $\mathrm{IIV}, V_{\mathrm{c}}(\mathrm{CV} \%)$ & 32.5 & 39.9 & 31.6 & $22.7-46.3$ \\
\hline IIV, $Q(\mathrm{CV} \%)$ & 24.8 & 100.0 & 28.4 & $0.134-45.4$ \\
\hline $\mathrm{IIV}, V_{\mathrm{p}}(\mathrm{CV} \%)$ & 22.4 & 54.8 & 22.2 & $15.2-32.6$ \\
\hline $\mathrm{IIV}, \mathrm{CL}_{n}(\mathrm{CV} \%)$ & 82.6 & 34.4 & 77.8 & $4.87-113$ \\
\hline Residual error for SCD-VOC (ng/mL) & 1.53 & 9.68 & 1.51 & $1.23-1.81$ \\
\hline Residual error for non-SCD-VOC (ng/mL) & 0.865 & 23.2 & 0.912 & $0.338-6.70$ \\
\hline$\sigma$ residual error for SCD-VOC $(\mathrm{CV} \%)$ & 21.49 & 8.6 & 20.9 & $15.2-26.3$ \\
\hline$\sigma$ residual error for non-SCD-VOC (CV\%) & 9.71 & 24.9 & 9.65 & $9.0-10.3$ \\
\hline$\sigma$ residual error for urine data $(\mathrm{CV} \%)$ & 31.1 & 23.9 & 30.7 & $24.8-38.5$ \\
\hline
\end{tabular}

Estimated parameters from final model using all studies, except B5201002

$C I$ confidence interval, $C L$ renal clearance, $C L_{n}$ nonrenal clearance, $\mathrm{CrCl}$ creatinine clearance, $C V \%$ coefficient of variance $\%, I I V$ inter-individual variability (IIV values represent the square root of variance $[\times 100]$ of the IIV parameter), non-SCD-VOC subjects without sickle cell disease or subjects with stable sickle cell disease not experiencing a vaso-occlusive crisis, $S C D$-VOC subjects with sickle cell disease experiencing a vaso-occlusive crisis, $Q$ inter-compartmental clearance, $V_{c}$ central volume of distribution, $V_{p}$ peripheral volume of distribution

subject, but it matches with the earlier reported value for this cohort [10].

As any missing observations in either urine volume or drug concentration affected every subsequent calculation of total amount eliminated in urine, the excretion rates for each collection period were also calculated because their calculation for one collection period was independent of another collection period. As seen from Fig. 4 of the ESM, the model-predicted excretion rates match very well with the values calculated from the observed data.

Derived PK metrics, such as $C_{\text {avg,ss }}$, were also calculated from the empirical Bayesian estimates of CL, obtained from the post hoc estimates for the phase III study (B5201002). Specifically, $C_{\text {avg.ss }}$ was calculated according to the formula:

$C_{\mathrm{avg} \mathrm{ss}, i}=\frac{1}{\tau} \times \frac{\text { Dose }_{i}}{\mathrm{CL}_{i}}$,

where $\tau$ is the inter-dosing interval and Dose ${ }_{i}$ and $\mathrm{CL}_{i}$ are the individual dose and Bayesian estimate of individual $\mathrm{CL}$, respectively. A comparison of the $C_{\mathrm{avg}, \mathrm{ss}}$ for the two cohorts of the phase III study is shown in Fig. 3, where the circles represent individual $C_{\text {avg,ss }}$ values and the box plot provides a summary of the observed $\mathrm{C}_{\mathrm{avg}, \mathrm{ss}}$ values. As seen from the figure, $C_{\text {avg,ss }}$ values were similar across the two cohorts in the phase III study (Fig. 3a) and were also similar when Cohort 1 was divided into adult and pediatric strata (Fig. 3b). More importantly, only a few observations were found to be below the $C_{\text {avg.ss }}$ value of $30 \mu \mathrm{g} / \mathrm{mL}$, the median $C_{\text {avg,ss }}$ for the lower dose of the phase II study (B5201012). Even fewer observations were below the minimum threshold of $10 \mu \mathrm{g} / \mathrm{mL}$. The median $C_{\text {avg.ss }}$ was observed to be within $42.65-46.20 \mu \mathrm{g} / \mathrm{mL}$, the median maximum concentration was observed to be within a range of $208.31-276.01 \mu \mathrm{g} / \mathrm{mL}$, and the median minimum concentration was observed to be within a range of $11.24-20.10 \mu \mathrm{g} / \mathrm{mL}$ for the two cohorts.

A key objective of the population PK analysis was to assess the similarity of exposures between the two cohorts of the phase III study (B5201002). Similarity of exposures was adjudicated based on a predefined criterion where the exposures were assumed to be similar if $80 \%$ of the exposures in Cohort 2 (subjects aged 6-11 years) were found to be between the 5th and 95th percentile of the exposure values in Cohort 1 (subjects aged $\geq 12$ years). Figure 4 shows a cumulative distribution function overlay of the exposures in the two cohorts. As seen in the figure, approximately $91.5 \%$ of the exposure values 


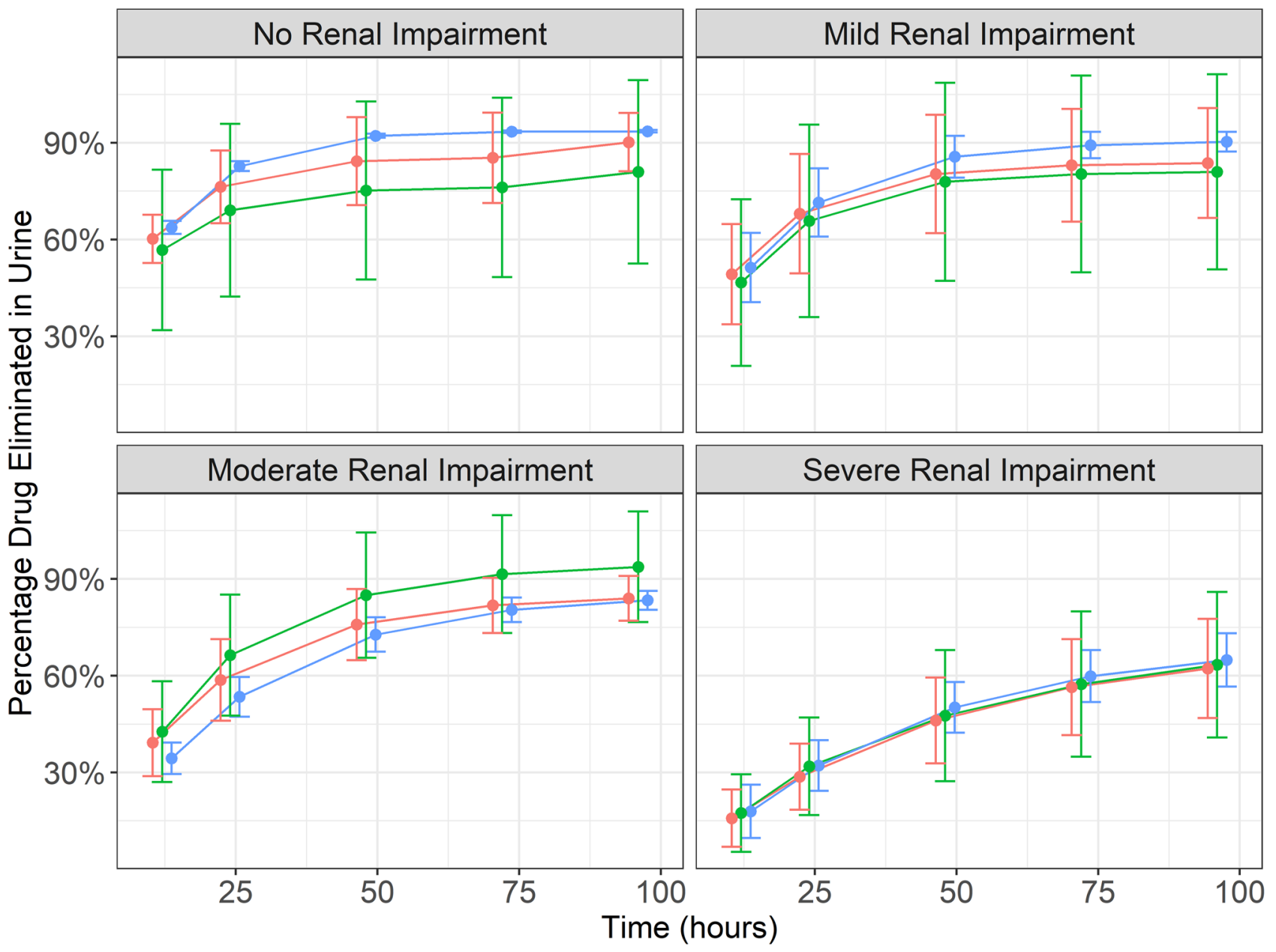

Fig. 2 Percentage of drug cleared in the phase I study in subjects with varying degrees of renal impairment. Each panel shows the mean and 1 standard deviation of observed data (green lines), the mean population prediction from the model (red lines), and mean individual pre-

dictions of cumulative drug eliminated in urine (blue lines) for subjects with varying degrees of renal impairment from the phase I study in renally impaired subjects (Study B5201005)

in Cohort 2 were between the predefined 5th and 95th percentiles of the values in Cohort 1 . This result provides us with confidence that the plasma drug exposure was similar between Cohort 1 and Cohort 2 .

To support dosing in renally impaired subjects, a simulated population of subjects was constructed and categorized into normal, mild, moderate, and severely renally impaired subsets, based on $\mathrm{CrCl}$ values obtained from the CKD-EPI formula. Height and weight for these subjects were simulated from the demographics reported in the phase III study. The simulations showed a clear correlation between renal impairment and PK parameters (CL) as well as derived PK parameters, such as $C_{\text {avg,ss }}, C_{\text {max,ss }}$, and total area under the concentration-time curve (Table 1 of the ESM). A wide variability was observed in $C_{\text {avg,ss }}$ values, with the median values of $C_{\text {avg,ss }}$ being approximately $100 \mu \mathrm{g} / \mathrm{mL}$ and $180 \mu \mathrm{g} / \mathrm{mL}$ for moderately and severely renally impaired subjects, respectively.

Similarly, to understand the effect of age in the model, a simulated population of pediatric subjects with demographics similar to subjects in the phase III study (B5201002) was assessed. Figure 5 shows a comparison between observed and simulated $C_{\text {avg,ss }}$, stratified by age. As seen in the figure, with lower age, a lower predicted/simulated concentration range is projected from the model. This could be due to the fact that with lower age, the $\mathrm{mg} / \mathrm{kg}$ dose does not correct entirely for the scaling of exposure with $\mathrm{CrCl}$.

Finally, using the population estimates from Table 3, and using the formula provided in Sect. 2.7, we calculated the terminal half-life of rivipansel to be about $7.5 \mathrm{~h}$. This value matches well with a previously calculated estimate of $7-8 \mathrm{~h}$. 


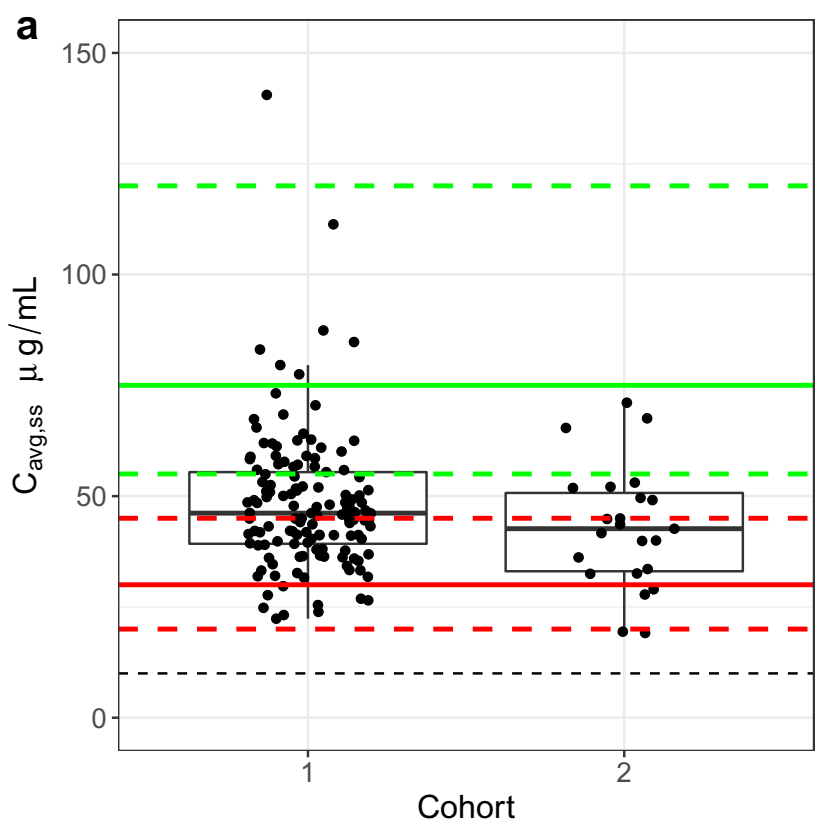

Fig. 3 Average steady-state concentration in the phase III study. For the phase III study data shown in this figure, Cohort 1 represents subjects who were aged 12 years or older, Cohort 2 represents subjects in the 6-11 years of age range (panel a). The two different strata of Cohort 1 are shown in panel $\mathbf{b}$. The black dashed horizontal line indicates the minimum target concentration of $10 \mu \mathrm{g} / \mathrm{mL}$. Solid red

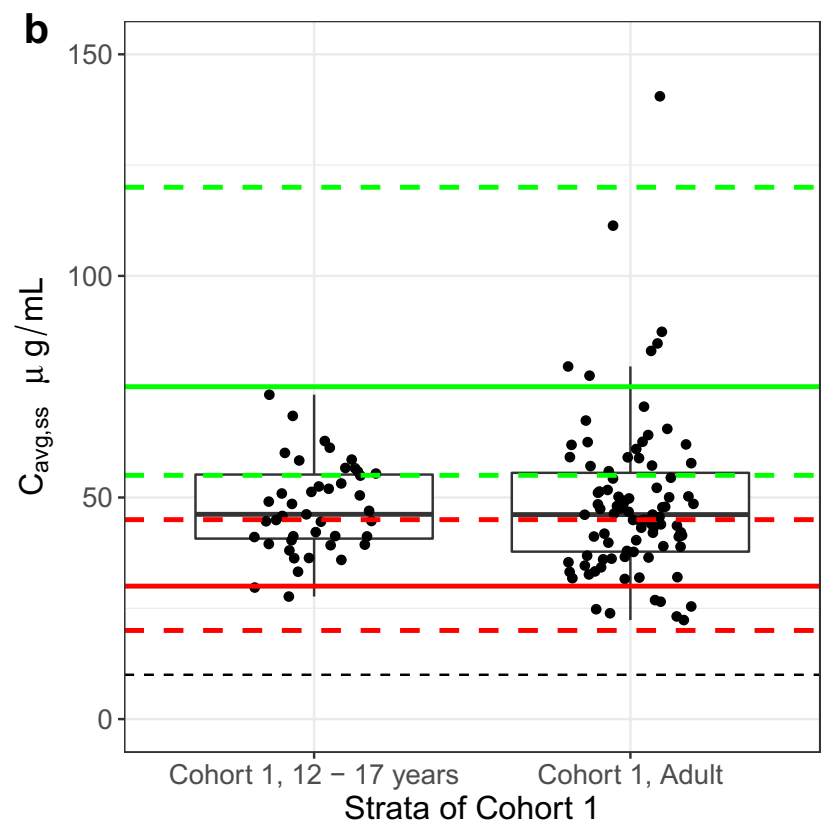

and green lines indicate the median concentration of the low dose $(30 \mu \mathrm{g} / \mathrm{mL})$ and the high dose $(75 \mu \mathrm{g} / \mathrm{mL})$, respectively, and dashed red and green lines indicate the upper and lower limits of the $95 \%$ prediction intervals of the low and high dose respectively, obtained from the phase II study (B5201012)
Fig. 4 Cumulative distribution function overlay of the exposures in the two phase III study cohorts (B5201002). The red and teal lines depict the cumulative frequency distribution of the fraction of patients with drug concentration (in $\mu \mathrm{g} / \mathrm{mL}$ ) on the $x$-axis. The blue vertical dashed lines represent 30 and $75 \mu \mathrm{g} / \mathrm{mL}$ concentrations, which are the median concentrations of the low dose and high dose of the phase II study respectively. The gray horizontal dashed lines are the guidelines representing the 5th and 95th percentiles

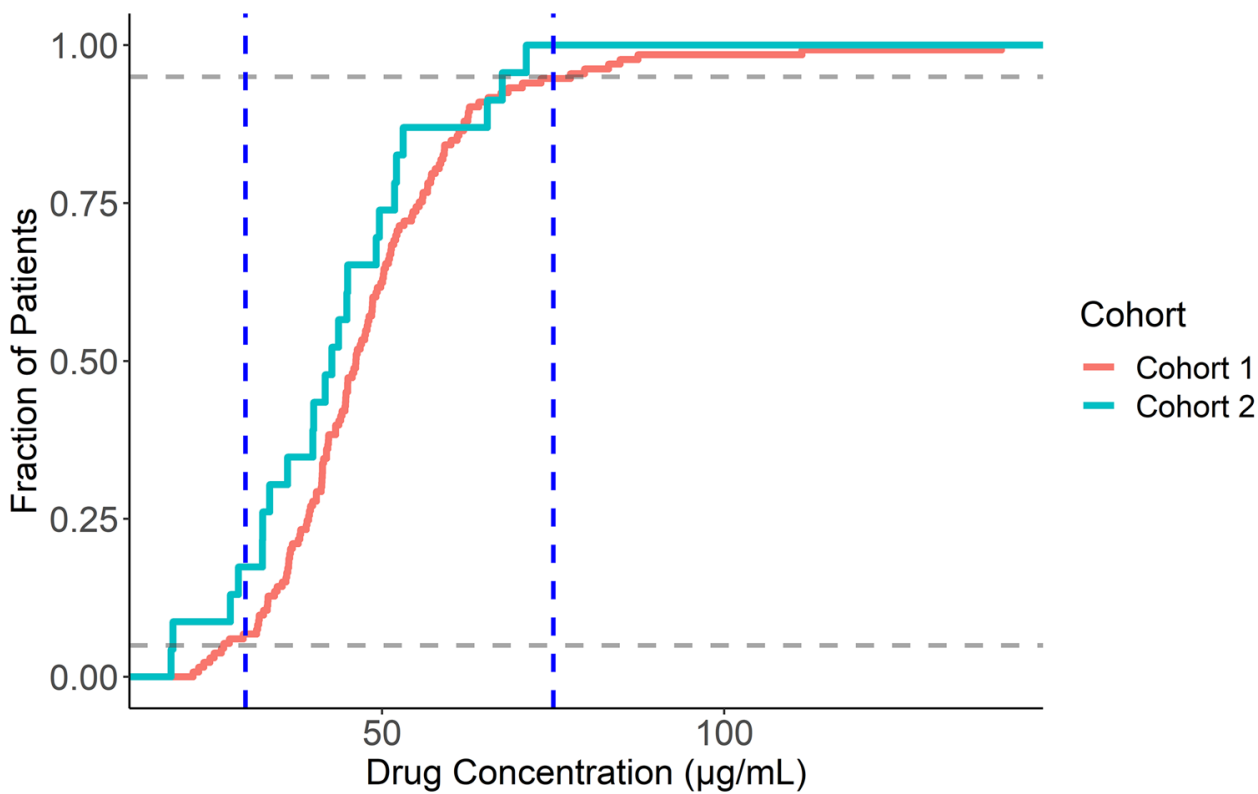

\section{Discussion}

At the time these studies were conducted, rivipansel was in development as a therapy to treat patients with SCD experiencing painful VOCs. Here, we provide a population
PK analysis of all previously conducted studies of rivipansel in healthy subjects and in stable subjects with SCD, including those experiencing an active VOC (Table 1). This modeling analysis extends the work done previously by Tammara and Harnisch [11], which modeled plasma 


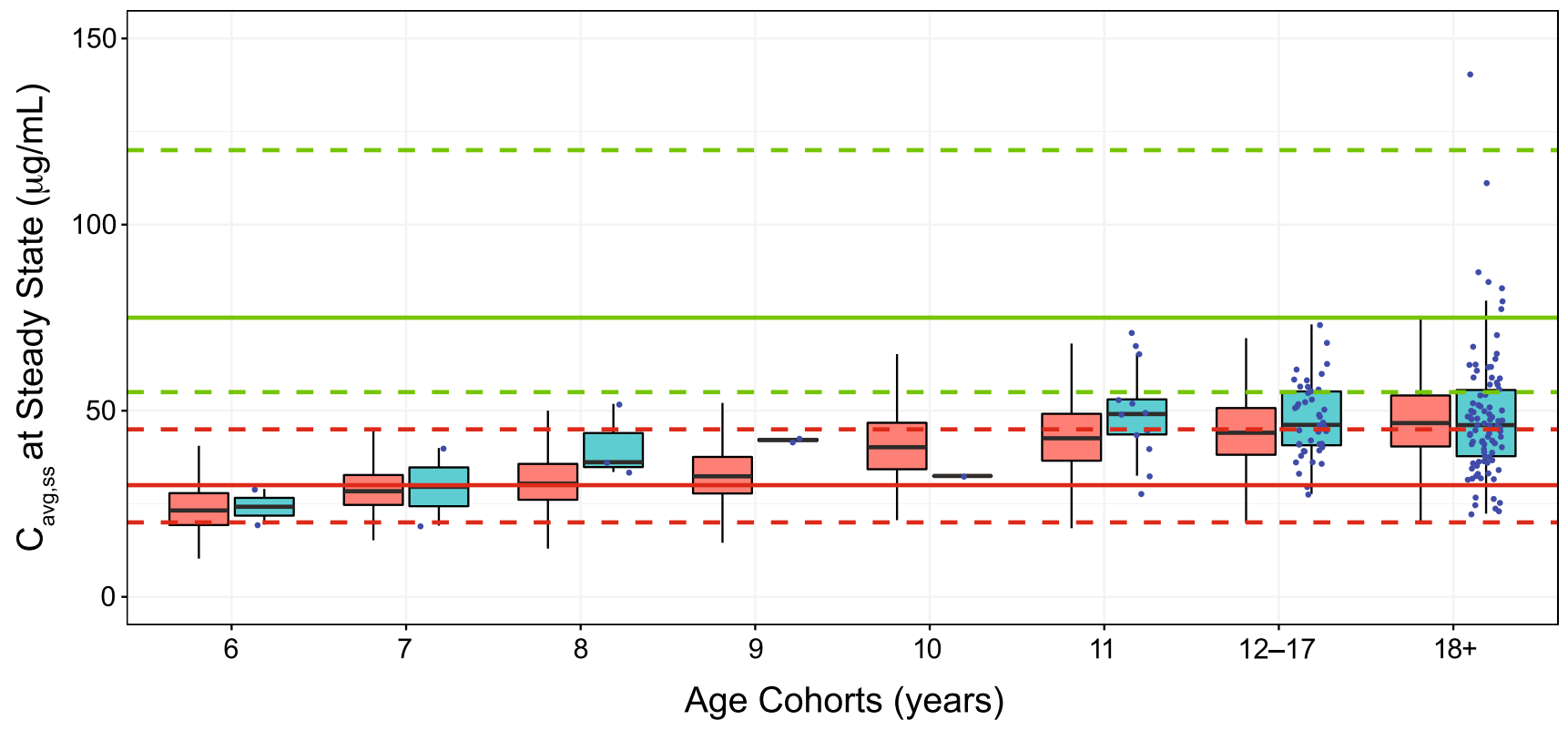

Fig. 5 Comparison of observed and simulated average steady-state concentration $\left(C_{\text {avg,ss }}\right)$ stratified by age. Red box plots show the range of values obtained from the simulation. Teal box plots show a summary of the post hoc estimated $C_{\text {avg.ss }}$ values and blue circles show the individual observed $C_{\text {avg,ss }}$ values from the phase III study. Solid

drug concentrations from three phase I studies and one phase II study using a three-compartment model to inform dosing recommendations for patients aged 6-11 years participating in the phase III study of rivipansel. Final parameter estimates for the rate of CL of rivipansel were slightly lower in the current analysis $(1.15 \mathrm{~L} / \mathrm{h})$ compared with the previous exercise $(1.25 \mathrm{~L} / \mathrm{h})$, as was the peripheral volume of drug distribution ( $4.48 \mathrm{~L}$ vs $4.68 \mathrm{~L})$, while the estimated central volume of distribution was higher in the current analysis (6.75 L vs $6.24 \mathrm{~L})$.

In this population PK analysis, we included plasma and urine data from all of the phase I studies, the phase II study, and studies conducted by Pfizer in special populations, such as renally or hepatically impaired subjects and subjects in different age categories, to provide a comprehensive picture of the PK profile of rivipansel. Our modeling analysis corroborates the almost exclusive elimination of the drug via the kidneys, as previously observed. The population estimated renal CL as a fraction of total CL was 0.94, and the model-predicted amount of drug excreted in urine matched well with clinical observations in the four studies in which urine data were collected. As rivipansel is cleared almost exclusively renally, the excretion rate has a potential to be used as a marker for renal CL. Our modeling also shows that the excretion rate plots were better than the cumulative elimination plots, as the excretion rate in one interval is unaffected by missing data in other intervals. red and green lines indicate the median concentration of the low dose $(30 \mu \mathrm{g} / \mathrm{mL})$ and the high dose $(75 \mu \mathrm{g} / \mathrm{mL})$, respectively, and dashed red and green lines indicate the upper and lower limits of the $95 \%$ prediction intervals of the low and high dose, respectively, of the phase II study (B5201012)

In the context of the phase III RESET study, the population PK model provided a novel framework to compare exposures between the two study cohorts. The findings from the phase III study population PK modeling suggest that dose adjustments of rivipansel may have been required in renally impaired patients and in pediatric patients.

A comparison of different formulae to estimate the glomerular filtration rate in our analysis showed that the CKDEPI formula for adults and the bedside Schwartz formula for children provided the best fit model, although we had to include an additional factor for subjects with a low estimated glomerular filtration rate to evaluate the renal CL of rivipansel. We tried an alternative approach by including serum creatinine and the various components to the different formulae as covariates in the model individually, which did not lead to an improvement in the model.

Subjects with SCD are known to have a greater degree of renal filtration than healthy adults, known as hyperfiltration; thus, it is important to quantify the higher CL observed in subjects with SCD. Our analysis showed that this hyperfiltration was approximately $12 \%$, which is lower than the previously calculated value from the modeling conducted with phase I studies only. Thus, we found that the degree of hyperfiltration may change, depending on the composition of the study population, but our population PK model provides a framework to calculate the degree of hyperfiltration for future studies in subjects with SCD. In conclusion, the population pharmacokinetics of 
rivipansel was well described by a two-compartment model with CL having a renal and a non-renal component. The renal CL was modeled to be dependent on $\mathrm{CrCl}$, age, and an additional factor for subjects with SCD undergoing a VOC. Our modeling analysis also corroborated the almost exclusive renal elimination of rivipansel and showed that derived PK estimates $\left(C_{\text {avg.ss }}\right)$ were similar between the pediatric (aged 6-11 years) subjects and subjects aged $\geq 12$ years in the pivotal phase III study.

Supplementary Information The online version contains supplementary material available at https://doi.org/10.1007/s40268-021-00346-3.

Acknowledgements Editorial support was provided by Michael D. Morren, RPh, MBA, of Peloton Advantage, LLC, an OPEN Health company, and funded by Pfizer Inc.

\section{Declarations}

Funding This study was funded by Pfizer Inc.

Conflict of Interest Satyaprakash Nayak, Brinda Tammara, and Lutz O. Harnisch are employees of Pfizer and may own stock/options in the company.

Ethics Approval All studies included in this analysis were approved by an institutional review board or ethics committee at each study center and were conducted in accordance with the principles of the Declaration of Helsinki, the International Council for Harmonisation Good Clinical Practice guidelines, and local regulatory requirements.

Consent to Participate In all studies, all patients provided written informed consent prior to participation.

Availability of Data and Material Upon request, and subject to certain criteria, conditions, and exceptions (see https://www.pfizer.com/scien ce/clinical-trials/trial-data-and-results for more information), Pfizer will provide access to individual de-identified participant data from Pfizer-sponsored global interventional clinical studies conducted for medicines, vaccines, and medical devices (1) for indications that have been approved in the USA and/or European Union or (2) in programs that have been terminated (i.e., development for all indications has been discontinued). Pfizer will also consider requests for the protocol, data dictionary, and statistical analysis plan. Data may be requested from Pfizer trials 24 months after study completion. The de-identified participant data will be made available to researchers whose proposals meet the research criteria and other conditions, and for which an exception does not apply, via a secure portal. To gain access, data requestors must enter into a data access agreement with Pfizer.

Author Contributions All authors contributed to the study conception and design. All authors collaborated in the preparation of the manuscript, with editorial support funded by Pfizer Inc., and critically reviewed and provided revisions to the manuscript. All authors had access to the data and assume responsibility for the completeness and accuracy of the data and data analyses. All authors granted final approval of the manuscript for submission.

Open Access This article is licensed under a Creative Commons Attribution-NonCommercial 4.0 International License, which permits any non-commercial use, sharing, adaptation, distribution and reproduction in any medium or format, as long as you give appropriate credit to the original author(s) and the source, provide a link to the Creative Commons licence, and indicate if changes were made. The images or other third party material in this article are included in the article's Creative Commons licence, unless indicated otherwise in a credit line to the material. If material is not included in the article's Creative Commons licence and your intended use is not permitted by statutory regulation or exceeds the permitted use, you will need to obtain permission directly from the copyright holder. To view a copy of this licence, visit http://creativecommons.org/licenses/by-nc/4.0/.

\section{References}

1. Centers for Disease Control and Prevention. Data and statistics on sickle cell disease (SCD). 2019. https://www.cdc.gov/ncbddd/ sicklecell/data.html. Accessed 21 Jul 2020.

2. Davis H, Moore JRRM, Gergen PJ. Cost of hospitalizations associated with sickle cell disease in the United States. Public Health Rep. 1997;112:40-3

3. Inusa BPD, Stewart CE, Mathurin-Charles S, et al. Paediatric to adult transition care for patients with sickle cell disease: a global perspective. Lancet Haematol. 2020;7:e329-41.

4. Kanter J, Kruse-Jarres R. Management of sickle cell disease from childhood through adulthood. Blood Rev. 2013;27:279-87.

5. Platt OS, Brambilla DJ, Rosse WF, et al. Mortality in sickle cell disease: life expectancy and risk factors for early death. N Engl J Med. 1994;330:1639-44.

6. Saraf SL, Molokie RE, Nouraie M, et al. Differences in the clinical and genotypic presentation of sickle cell disease around the world. Paediatr Respir Rev. 2014;15:4-12.

7. Manwani D, Frenette PS. Vaso-occlusion in sickle cell disease: pathophysiology and novel targeted therapies. Blood. 2013;122:3892-8.

8. Ware RE, de Montalembert M, Tshilolo L, Abboud MR. Sickle cell disease. Lancet. 2017;390:311-23.

9. Etzioni A. Adhesion molecules: their role in health and disease. Pediatr Res. 1996;39:191-8.

10. Tammara BK, Ryan K, Plotka A, et al. Effect of renal or hepatic impairment on the pharmacokinetics, safety, and tolerability of intravenous rivipansel. Clin Pharmacol Drug Dev. 2020;9:918-28.

11. Tammara BK, Harnisch LO. Dose selection based on modeling and simulation for rivipansel in pediatric patients aged 6 to 11 years with sickle cell disease. CPT Pharmacomet Syst Pharmacol. 2017;6:845-54

12. Telen MJ, Wun T, McCavit TL, et al. Randomized phase 2 study of GMI-1070 in SCD: reduction in time to resolution of vaso-occlusive events and decreased opioid use. Blood. 2015;125:2656-64.

13. de Paula RP, Nascimento AF, Sousa SM, Bastos PR, Barbosa AA. Glomerular filtration rate is altered in children with sickle cell disease: a comparison between Hb SS and Hb SC. Rev Bras Hematol Hemoter. 2013;35:349-51.

14. Haymann JP, Stankovic K, Levy P, et al. Glomerular hyperfiltration in adult sickle cell anemia: a frequent hemolysis associated feature. Clin J Am Soc Nephrol. 2010;5:756-61.

15. Audard V, Bartolucci P, Stehle T. Sickle cell disease and albuminuria: recent advances in our understanding of sickle cell nephropathy. Clin Kidney J. 2017;10:475-8.

16. Ware RE, Rees RC, Sarnaik SA, et al. Renal function in infants with sickle cell anemia: baseline data from the BABY HUG trial. J Pediatr. 2010;156:66-70.

17. Aygun B, Mortier NA, Smeltzer MP, Shulkin BL, Hankins JS, Ware RE. Hydroxyurea treatment decreases glomerular hyperfiltration in children with sickle cell anemia. Am J Hematol. 2013;88:116-9. 
18. Dampier CD, Telen MJ, Wun T, et al. Efficacy and safety of rivipansel (GMI-1070) in the treatment of vaso-occlusive crisis in hospitalized patients with sickle cell disease: results from the RESET phase 3 study [abstract]. Presented at: Annual Sickle Cell Disease Research and Educational Symposium and National Sickle Cell Disease Scientific Meeting; 23-25 September 2020.

19. Tammara BK, Plotka A, Shafer FE, Readett DRJ, Riley S, KorthBradley JM. Lack of effect of rivipansel on QTc interval in healthy adult African American male subjects. J Clin Pharmacol. 2017;57:1315-21.

20. Wun T, Styles L, DeCastro L, et al. Phase 1 study of the E-selectin inhibitor GMI 1070 in patients with sickle cell anemia. PLoS ONE. 2014;9:e101301.

21. Levey AS, Stevens LA, Schmid CH, et al. A new equation to estimate glomerular filtration rate. Ann Intern Med. 2009;150:604-12.

22. Schwartz GJ, Munoz A, Schneider MF, et al. New equations to estimate GFR in children with CKD. J Am Soc Nephrol. 2009;20:629-37.
23. DuBois D, DuBois EF. Clinical calorimetry tenth paper a formula to estimate the approximate surface area if height and weight be known. Arch Intern Med (Chic). 1916;17:863-71.

24. Levey AS, Coresh J, Greene T, et al. Expressing the Modification of Diet in Renal Disease Study equation for estimating glomerular filtration rate with standardized serum creatinine values. Clin Chem. 2007;53:766-72.

25. Cockcroft DW, Gault MH. Prediction of creatinine clearance from serum creatinine. Nephron. 1976;16:31-41.

26. Bauer RJ. NONMEM tutorial part II: estimation methods and advanced examples. CPT Pharmacomet Syst Pharmacol. 2019;8:538-56.

27. Toutain PL, Bousquet-Melou A. Plasma terminal half-life. J Vet Pharmacol Ther. 2004;27:427-39.

28. Bauer RJ. NONMEM users guide: introduction to NONMEM 7.4.1. Gaithersburg (MD): ICON Development Soluitons; 2017.

29. R Core Team. R: a language and environment for statistical computing. R Foundation for Statistical Computing, Vienna. 2020. https://www.r-project.org/. Accessed 5 May 2020. 